\title{
MicroRNA-148b targets Rho-associated protein kinase 1 to inhibit cell proliferation, migration and invasion in hepatocellular carcinoma
}

\author{
${\text { XIAN } \mathrm{CHEN}^{1}{ }^{1} \text {, LIANHUA BO }}^{1}$, WEI LU $^{1}$, GUIHUA ZHOU $^{1}$ and QI CHEN $^{1,2}$ \\ Departments of ${ }^{1}$ Infectious Diseases and ${ }^{2}$ Urology, The Fourth Affiliated Hospital of Nantong Medical College, \\ Yancheng City No. 1 People's Hospital, Yancheng, Jiangsu 224001, P.R. China
}

Received January 1, 2015; Accepted September 25, 2015

DOI: $10.3892 / \mathrm{mmr} .2015 .4500$

\begin{abstract}
RNA(miR)-148b has been found to be downregulated in various human malignancies, including hepatocellular carcinoma (HCC) as well as gastric, pancreatic, colon and oral cancer. However, the function of miR-148b in $\mathrm{HCC}$ has remained elusive. The present study examined the effects of miR-148b on the proliferation, migration and invasion of HCC cells in vitro. After transfection of the HepG2 and SMMC-7721 HCC cell lines with miR-148b, an MTT assay, a Transwell migration and invasion assay as well as western blot analysis were performed. miR-148b was shown to inhibit cell proliferation, migration and invasion in the two cell lines. Using a luciferase reporter assay, the present study also provided the first evidence that miR-148b directly targets Rho-associated protein kinase 1 in HCC. These results suggested that miR-148 may represent a novel molecular marker and a potential molecular therapeutic for inhibiting metastasis of HCC.
\end{abstract}

\section{Introduction}

As one of the most common types of malignant liver tumors, hepatocellular carcinoma (HCC) is the sixth most common malignancy and the third most prevalent cause of cancer-associated mortality worldwide (1). Of note, the number of Chinese patients with HCC accounts for $>50 \%$ of global cases of HCC (2). Each year, an estimate of 21,000 new diagnoses of HCC are posed and 700,000 HCC-associated mortalities occur (3). As all neoplastic malignancies, HCC is generated via a complex, multi-step process comprising various genetic and

Correspondence to: Professor Qi Chen, Department of Urology, The Fourth Affiliated Hospital of Nantong Medical College, Yancheng City No. 1 People's Hospital, 15 Yuehe Road, Yancheng, Jiangsu 224001, P.R. China

E-mail: wudeyao_850405@163.com

Key words: microRNA-148b, Rho-associated protein kinase 1, hepatocellular carcinoma, metastasis epigenetic aberrations which affect a variety of and distinct etiology-specific pathways (4). Chronic inflammation, multiple viral infections, obesity, chronic alcohol abuse and diabetes are known to be risk factors for $\operatorname{HCC}(5,6)$. In most cases, current treatment strategies, including hepatectomy or orthotopic liver transplantation, significantly improve the patient's quality of life. However, the overall prognosis remains poor for the majority of HCC patients $(7,8)$. Of note, fatal tumor recurrence or metastasis occur in as many as $50 \%$ at two years and $75-100 \%$ at five years after surgery (9). In spite of recent advances in functional genomics, which have enhanced the understanding of hepatocarcinogenesis, the molecular pathogenesis of HCC has largely remained elusive $(10,11)$. It is therefore required to clarify the underlying mechanisms of HCC, which may lead to the development of improved prognostic tools, identification of novel therapeutic targets and approaches to suppress cancer recurrence.

MicroRNAs (miRNAs/miRs) are non-coding, endogenous, single-stranded RNA molecules of 22 nucleotides in length, which regulate human gene expression (12). At present, it is thought that miRNAs potentially regulate almost one third of all coding genes in the human genome (13). miRNAs generally bind to the 3'-untranslated region (UTR) of target mRNAs through base pairing (14). In recent years, accumulating evidence has suggested a functional contribution of specific miRNAs in diverse biological processes $(15,16)$, including the de-regulation of miRNAs by acting on their targeted genes during the progression and tumorigenesis of human cancers $(17,18)$. In addition, an increasing number of studies have demonstrated the differential expression of miRNAs in various cell types and developmental stages, indicating that miRNAs regulate a variety of biological functions and disease-associated processes, including cell proliferation, differentiation, metabolism, programmed cell death and metastasis (19,20). Furthermore, miRNAs have been demonstrated to be frequently located in genomic breakpoint regions in human cancers and to act as either oncogenes or tumor suppressor genes during tumorigenesis and cancer progression (21).

miR-148b has been shown to be significantly reduced in HCC (22). The present investigated the potential role of miR-148b in HCC cells. Therefore, miR-148b was transfected 
into HCC cells and its effect on proliferation, migration and invasion were assessed. Furthermore, the present study aimed to identify direct target genes of miR-148b. The present study showed that miR-148b inhibited cell proliferation, migration and invasion in the HepG2 and SMMC-7721 HCC cell lines, indicating a tumor-suppressive role of miR-148b in HCC. The present study also provided the first evidence that miR-148b directly targets Rho-associated protein kinase 1 (ROCK1) in HCC cells.

\section{Materials and methods}

Cell culture and transfection. The HepG2 and SMMC-7721 human HCC cell lines were obtained from the Shanghai Institute of Biochemistry and Cell Biology (Shanghai, China). Cells were cultured in RPMI-1640 medium (Gibco; Thermo Fisher Scientific, Inc., Waltham, MA, USA) supplemented with $10 \%$ heat-inactivated fetal bovine serum (FBS; Gibco; Thermo Fisher Scientific, Inc.) in a humidified atmosphere with $5 \% \mathrm{CO}_{2}$ at $37^{\circ} \mathrm{C}$.

The mature miR-148b mimics and negative control miRNA mimics (NC) were designed and synthesized by GenePharma (Shanghai, China). The sequence of the miR-148b mimic was as follows: 5'-UCAGUGCAUCA CAGAACUUUGU-3'. The sequence of NC mimic was 5'-UUCUCCGAACGUGUCACGUTT-3'. Prior to transfection, the cells were seeded in a 6-well plate and cultured in RPMI1640 medium without antibiotics. For functional analysis, the cells were transfected with the miR-148b or NC mimics using Lipofectamine ${ }^{\circledR} 2000$ (Invitrogen; Thermo Fisher Scientific, Inc.), according to the manufacturer's protocol.

Cell viability assay. Cell viability was assessed using a 3-(4, 5-dimethylthiazolyl-2-yl)-2-5 diphenyl tetrazolium bromide (MTT; $5 \mathrm{mg} / \mathrm{mL}$ ) assay. Cells transfected with miR-148b or NC were seeded onto 96-well plates at a density of 3,000 cells per well in a final volume of $100 \mu \mathrm{l}$. Following incubation for 24 , 48, 72, 96, 120 or 144 h, $20 \mu 1$ MTT (Sigma-Aldrich, St. Louis, MO, USA) was added to each well. The plates were incubated for $4 \mathrm{~h}$ at $37^{\circ} \mathrm{C}$ followed by replacement of the medium with $200 \mu \mathrm{l}$ dimethyl sulfoxide (Sigma-Aldrich). The absorbance at was measured as the optical density (OD) at $490 \mathrm{~nm}$ using an automatic multi-well spectrophotometer (Bio-Rad Laboratories, Inc., Hercules, CA, USA). The growth inhibition rate was calculated using the following formula: Inhibitory rate $=\left(1-\mathrm{OD}_{\text {miR-148b }} / \mathrm{OD}_{\text {miR-NC }}\right) \times 100 \%$. Three independent experiments were performed with three replicates in each.

Cell migration and invasion assay. Cell migration and invasion assays were performed in a 24 -well Boyden chamber with an $8-\mu \mathrm{m}$ pore-size polycarbonate membrane (Corning-Costar, Corning, NY, USA). Cells $\left(1 \times 10^{5} /\right.$ well $)$ transfected with miR-148b or NC in $200 \mu \mathrm{l}$ serum-free RPMI-1640 were seeded into the upper wells of the Transwell compartments with or without Matrigel (BD Biosciences, Franklin Lakes, NJ, USA) coating for the migration and invasion assays, respectively, while $500 \mu \mathrm{l}$ medium containing $20 \%$ FBS was added to the lower chamber. Incubation was performed for 12 and $24 \mathrm{~h}$ for the migration and the invasion assay, respectively. Subsequently, cells on the lower side of the membranes were fixed in $100 \%$ methanol (Beyotime Institute of Biotechnology, Haimen, China) for 2 min and cells on the upper side of the membranes were removed using cotton swabs. The migrated or invaded cells were stained with $0.5 \%$ crystal violet (Beyotime Institute of Biotechnology) for $2 \mathrm{~min}$ and images were captured using a light microscope (Olympus IX53; Olympus, Tokyo, Japan). Values for migration and invasion were obtained by counting five fields per membrane and represented as the average of three independent experiments Each experiment was performed at least three times, with each experimental condition repeated in triplicate.

Luciferase reporter assay. A target search for miR-148b using Targetscan (http://www.targetscan.org/) indicated that the 3'UTR of ROCK1 mRNA contains a potential targeted sequence for miR-148b. To experimentally confirm whether ROCK1 is a direct target of miR-148b, a luciferase reporter assay was performed. HepG2 or SMMC-7721 cells were seeded onto a 12 -well plate at $\sim 90 \%$ confluence and co-transfected with miR-148b or NC, and a luciferase reporter vector (GenePharma, Shanghai, China) containing the wild-type (WT) or mutant (Mut) 3'-UTR of ROCK1, using Lipofectamine 2000 (Invitrogen). Following $48 \mathrm{~h}$ of incubation, the cells were harvested and firefly luciferase activity was measured using the Dual Luciferase Assay system (Promega, Madison, WI, USA) with the results normalized to Renilla luciferase activity. Each assay was performed as three replicates.

Western blot analysis. Cells were harvested in radioimmunoprecipitation assay lysis buffer (Beyotime Institute of Biotechnology) at $72 \mathrm{~h}$ after transfection with miR-148b and NC. Following determination of the protein concentration using a bicinchoninic acid protein assay kit (Beyotime Institute of Biotechnology), equal quantities of protein $(20 \mu \mathrm{g})$ were separated by $10 \%$ SDS-PAGE (Beyotime Institute of Biotechnology) and electrotransferred onto polyvinylidene difluoride membranes (Beyotime Institute of Biotechnology). The membranes were blocked with 5\% non-fat milk, followed by incubation overnight at $4^{\circ} \mathrm{C}$ with the following primary antibodies: Rabbit anti-human monoclonal ROCK1 antibody (cat. no. 4035; 1:1,000 dilution; Cell Signaling Technology, Inc., Danvers, MA, USA) and rabbit anti-human monoclonal $\beta$ actin antibody (cat. no. 8546; 1:1,000 dilution; Cell Signaling Technology, Inc.). After washing with Tris-buffered saline (TBS) three times, incubation with the corresponding horseradish peroxidase-conjugated secondary antibody was performed for $1 \mathrm{~h}$. Following three washes with TBS, the bound secondary antibody was visualized using enhanced chemiluminescence solution (Pierce, Rockford, IL, USA) and images were captured using the FluorChem imaging system (Alpha Innotech, San Leandro, CA, USA). The protein intensities were quantified using AlphaEase FC software. (version 4.1.0; Alpha Innotech).

Statistical analysis. Values are expressed as the mean \pm standard deviation. Differences between groups were assessed using Student's $t$-test with Stata 10.0 software (Stata Corp., College Station, Texas, USA). A two-tailed P-value of less than 0.05 was considered to indicate a statistically significant difference between values. 


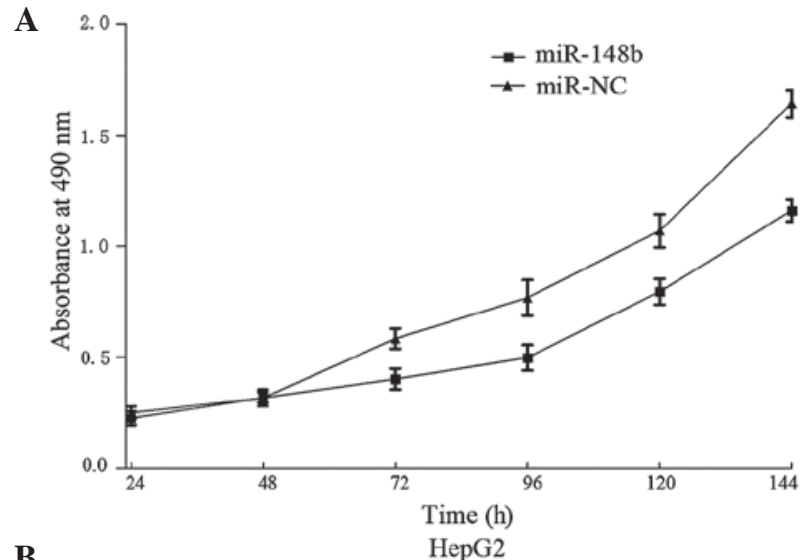

B

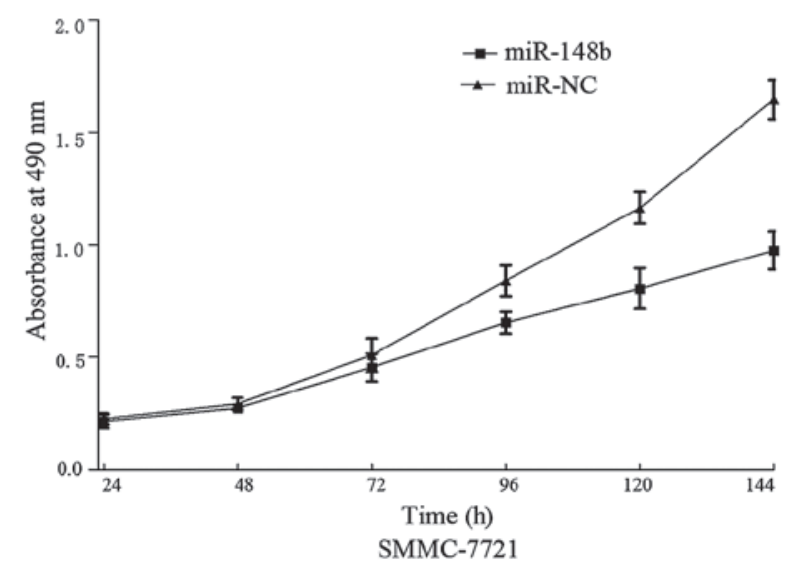

Figure 1. Viability of (A) HepG2 and (B) SMMC-7721 cells after transfection with miR-148b. Cell proliferation was determined using the MTT assay. Upregulation of miR-2148b significantly suppressed the proliferation of the hepatocellular carcinoma cell lines. Values are expressed as the mean \pm standard deviation. miR, microRNA; NC, negative control.

\section{Results}

miR $-148 b$ reduces the proliferation of HCC cell lines. To assess the effects of miR-148b on cell proliferation, an MTT assay was used. As expected, upregulation of miR-148b significantly inhibited the proliferation of the HepG2 and SMMC-7721 cell lines (Fig. 1; P<0.05). Following $144 \mathrm{~h}$ of treatment, the suppression rate of $\mathrm{miR}-148 \mathrm{~b}$ reached $29.29 \pm 2.8 \%$ in HepG2 cells and $41.82 \pm 3.5 \%$ in SMMC-7721 cells.

miR-148b inhibits the migration and invasion of HCC cell lines. To study the role of miR-148b in the regulation of invasion and migration of HCC cells, HepG2 or SMMC-7721 cells transfected with miR-148b or NC were cultured in Transwells with or without Matrigel, respectively. As shown in Fig. 2, transfection with miR-148b mimics significantly suppressed migration and invasion of HepG2 and SMMC-7721 cells $(\mathrm{P}<0.05)$. These results indicated that miR-148b suppressed the migration and invasion of HCC cells.

ROCK1 is a direct target gene of miR-148b in HCC. To identify targets of miR-148b in HCC, a target search using Targetscan was performed. ROCK1 was predicted to be a target of miR-148b, as the 3'UTR of its mRNA contains a
A

\section{Migration}
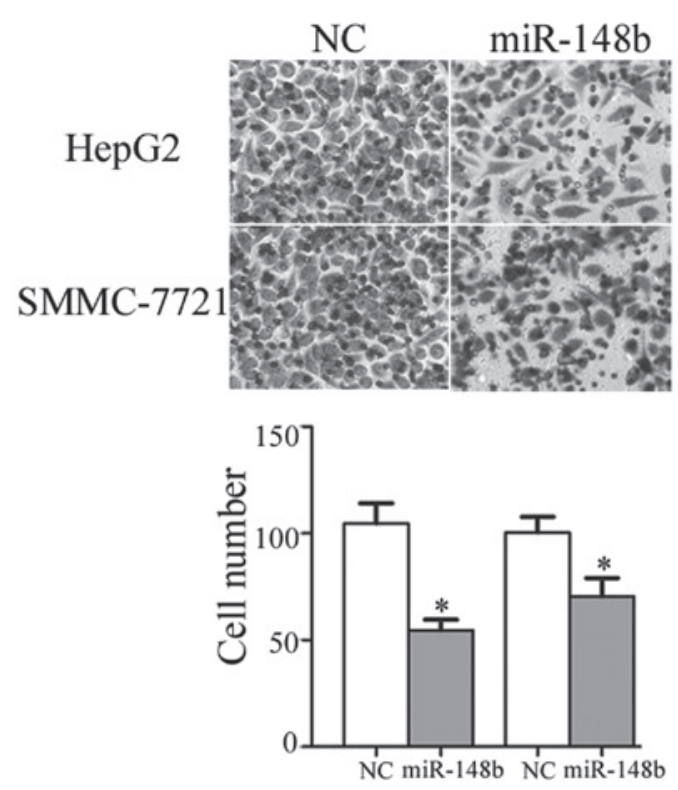

B

\section{Invasion}
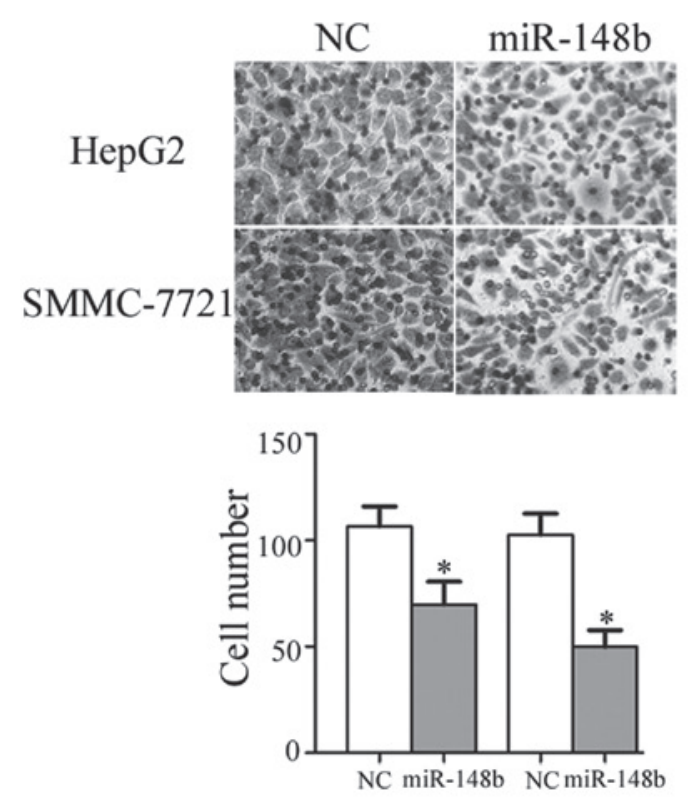

Figure 2. Inhibition of tumor cell migration and invasion by miR-148b in hepatocellular carcinoma cell lines. Transwell assays showed that (A) cell migration and (B) invasion were significantly decreased in miR-148b-transfected groups compared with those in the control group. Magnification, x200. Values are expressed as the mean \pm standard deviation. " $\mathrm{P}<0.05$, compared with the respective control. miR, microRNA; NC, negative control.

region with affinity for miR-148b (Fig. 3A). To verify whether miR-148b directly targets ROCK1, a luciferase reporter assay was performed. miR-148b was shown to significantly inhibit luciferase activity of a reporter vector containing the WT sequence of the ROCK1 3'UTR targeted by miR-148b, while luciferase activity of the reporter vector containing the Mut sequence was not affected by miR-148b in HepG2 and SMMC-7721 cells (Fig. 3B; $\mathrm{P}<0.05$ ). 


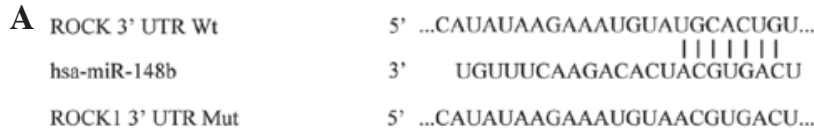

B

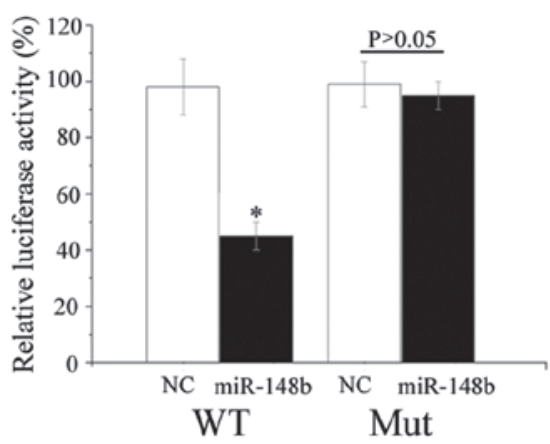

HepG2

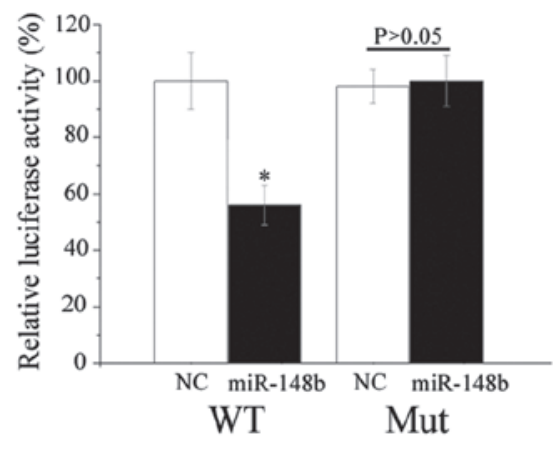

SMMC-7721
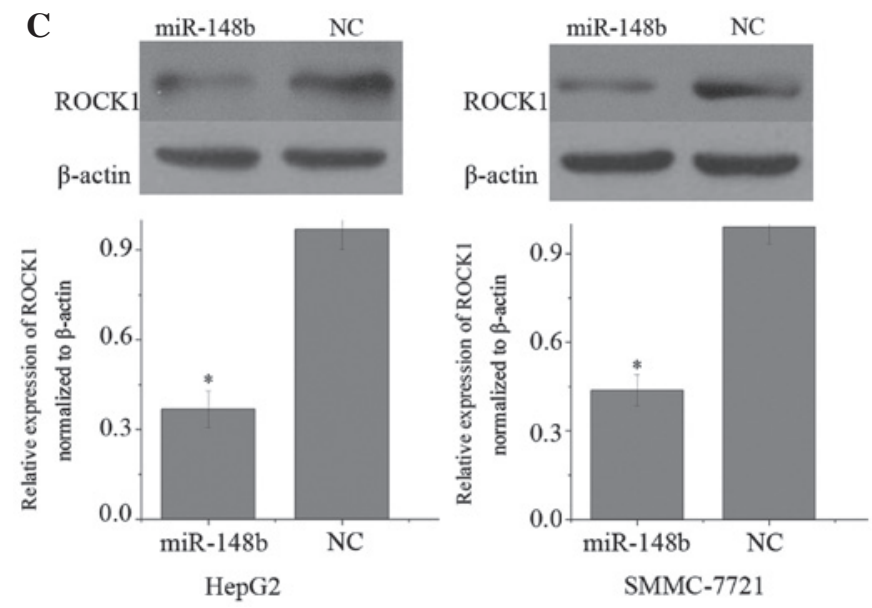

Figure 3. (A) Targetscan analysis indicated the presence of a seven-nucleotide seed match with miR-148b in the 3'UTR of ROCK1 mRNA at the 867-873 bp location. (B) A luciferase reporter assay demonstrated that ROCK1 is a direct target of miR-497 in vitro. miR-148b reduced the luciferase activity of a reporter plasmid containing the target sequence in the ROCK1 3'UTR in HepG2 and SMMC-7721, while the plasmid containing a mutated sequence was not affected. (C) ROCK1 protein expression was significantly reduced in HepG2 and SMMC-7721 cells after transfection with miR-148b. Values are expressed as the mean \pm standard deviation. ${ }^{*} \mathrm{P}<0.05$, compared with the respective control. miR, microRNA; NC, negative control; Mut, mutated; WT wild-type; ROCK1, Rho-associated protein kinase 1; UTR, untranslated region; hsa, Homo sapiens.

Furthermore, western blot analysis was performed to explore whether ROCK1 was decreased after transfection of HCC cell lines with miR-148b mimics. As shown in Fig. 3C,
ROCK1 was significantly downregulated in HepG2 and SMMC-7721 after transfection with miR-148b $(\mathrm{P}<0.05)$. All of these results provided in vitro evidence for ROCK1 being a direct target gene of miR-148b in HCC cells.

\section{Discussion}

miRNA-encoding genes represent $\sim 1 \%$ of the genome of various species and aberrant expression of miRNAs is known to be associated with numerous types of cancer (23). As miRNA expression can be easily tuned using molecular biology techniques, the upregulation of tumor-suppressive miRNAs and silencing of oncogenic miRNAs represents a promising strategy for cancer therapies (24). Numerous studies have suggested the use of miRNAs as targets or tools for anti-cancer therapy (25).

miR-148b is a member of the miR-148/152 family, which comprises miR-148a, miR-148b and miR-152 (26). Being 21-22 nucleotides in length, mature members of the miR-148/152 family have an identical seed sequence of 6-7 nucleotides, which binds to target mRNAs (27). miR-148b is located on chromosome 12 q13 and has been linked with numerous human malignancies; microarray analyses have demonstrated that miR-148b is frequently downregulated in various tumor types, including colorectal (28), gastric (29), pancreatic (30), colon (31) and oral cancer (32), as well as HCC (22).

Song et al (29) reported that in gastric cancer, the expression of miR-148b was associated with the tumor size and that ectopic expression of miR-148b inhibited cell proliferation by targeting cholecystokinin $\mathrm{B}$ receptor (CCKBR) in vitro and in vivo (29). The same group also reported that in colorectal cancer, miR-148b affected cell proliferation by regulating the expression of CCKBR, whose function is dependent on gastrin (28). In breast cancer, miR-148b was also shown to inhibit the invasion, resistance to anoikis, extravasation and lung metastasis formation of breast cancer cells (33). Furthermore, Wu et al (34) revealed that miR-148b increased the radiosensitivity of non-Hodgkin's lymphoma cells. These studies suggested that miR-148b serves as a potential tumor suppressor. Therefore, transfection with miR-148b mimics or administration of pharmaceutical compounds which cause upregulation of miR-148b expression are expected to be effective therapeutic strategies for cancer.

Previous studies have shown that the expression of miR-148b was decreased in HCC tissues compared with that in normal control specimens; in addition, miR-148b expression was shown to be closely associated with $\mathrm{HCC}$ vein invasion and the tumor-nodes-metastasis stage (22). However, the potential function of miR-148b in HCC had remained elusive. The present study revealed that in HCC, miR-148b may function as a tumor suppressor through repression of ROCK1. Transfection with miR-148b mimics resulted in decreased cell proliferation, migration and invasion in HCC cells. These results suggested that $\mathrm{miR}-148 \mathrm{~b}$ may represent a molecular marker and therapeutic tool to inhibit metastasis of HCC.

For the development of metastasis, enhanced motility and invasion of tumor cells are required for them to detach from the primary tumor and enter lymph or blood vessels to 
reach distant sites of the body (35). Invasion and metastasis of cancer cells are regulated by various molecular pathways, among which the ROCK signaling pathway has a major role (36). Overexpression of ROCK, the fundamental downstream effector of the small guanine triphosphatase Rho, enhances the invasion and metastasis of various solid tumor cell types, including hepatocellular (37), breast (38) and colon cancers (39). Inhibiting ROCK may therefore represent a therapeutic approach for these tumor types.

ROCK has been reported to exist as two isoforms, ROCK1 and ROCK2, which are highly homologous with $65 \%$ amino acid sequence homology. Furthermore, sequence analysis revealed a $92 \%$ similarity of their kinase domains $(40,41)$. Human ROCK1 is encoded by chromosome 18 (18q11.1) (42), has been associated with cancer progression and was shown to be overexpressed in several cancer types (43). In HCC, upregulated expression of ROCK1 was associated with cytoskeleton remodeling as well as the motility and invasiveness of $\mathrm{HCC}$ cells (44). Several studies revealed that ROCK1 functions as an oncogene and is regulated by a number of miRNAs in human cancers. In gastric cancer, Zheng et al (35) revealed that miR-148a suppressed cell invasion and metastasis by directly targeting ROCK1. In bladder cancer and glioma, miR-124 inhibited cell migration and invasion by downregulating the expression of ROCK1 $(45,46)$. ROCK1 was also targeted by miRNAs in other types of human cancer, including miR-584 in renal cell carcinoma (47), miR-335 in neuroblastoma (48) and miR-146a in prostate cancer (49). The present study revealed that miR-148b suppressed HCC cell proliferation, migration and invasion by directly targeting ROCK1. miR-148b may therefore be used as a biomarker for early detection of tumor recurrence and as a targeted therapeutic to inhibit the invasiveness and metastasis formation of HCC.

In conclusion, the present study is the first, to the best of our knowledge, to show that miR-148b inhibited the proliferation, migration and invasion of HCC cells through reducing ROCK1 expression. Through the identification of a novel target gene of miR-148b, the present study enhanced the current understanding of carcinogenic mechanisms in HCC. The findings of the present study may lead to the development of novel diagnostic and therapeutic approaches for HCC. Further investigation is required to identify additional target genes of miR-148b, and to assess its suitability for use in the treatment of HCC.

\section{References}

1. Fares N and Pèron JM: Epidemiology, natural history and risk factors of hepatocellular carcinoma. Rev Prat 63: 216-217, 2013 (In French).

2. Huitzil-Melendez FD, Capanu M, O'Reilly EM, Duffy A, Gansukh B, Saltz LL and Abou-Alfa GK: Advanced hepatocellular carcinoma: Which staging systems best predict prognosis? J Clin Oncol 28: 2889-2895, 2010.

3. Zou C, Li Y, Cao Y, Zhang J, Jiang J, Sheng Y, Wang S, Huang A and Tang H: Up-regulated MicroRNA-181a induces carcinogenesis in hepatitis B virus-related hepatocellular carcinoma by targeting E2F5. BMC Cancer 14: 97, 2014.

4. Giordano S and Columbano A: MicroRNAs: New tools for diagnosis, prognosis and therapy in hepatocellular carcinoma? Hepatology 57: 840-847, 2013.

5. El-Serag HB: Epidemiology of viral hepatitis and hepatocellular carcinoma. Gastroenterology 142: 1264-1273, 2012.
6. Sanyal AJ, Yoon SK and Lencioni R: The etiology of hepatocellular carcinoma and consequences for treatment. Oncologist 15 (Suppl 4): S14-S22, 2010.

7. Rahbari NN, Mehrabi A, Mollberg NM, Muller SA, Koch M, Buchler MW and Weitz J: Hepatocellular carcinoma: Current management and perspectives for the future. Ann Surg 253: 453-469, 2011.

8. Worns MA and Galle PR: Future perspectives in hepatocellular carcinoma. Dig Liver Dis 42 (Suppl 3): S302-S309, 2010.

9. Portolani N, Coniglio A, Ghidoni S, Giovanelli M, Benetti A Tiberio GA and Giulini SM: Early and late recurrence after liver resection for hepatocellular carcinoma: Prognostic and therapeutic implications. Ann Surg 243: 229-235, 2006.

10. Thorgeirsson SS, Lee JS and Grisham JW: Functional genomics of hepatocellular carcinoma. Hepatology 43 (2 Suppl 1): S145-S150, 2006.

11. Villanueva A, Newell P, Chiang DY, Friedman SL and Llovet JM: Genomics and signaling pathways in hepatocellular carcinoma. Semin Liver Dis 27: 55-76, 2007.

12. Wang MJ, Li Y, Wang R, Yu YY, Yang L, Zhang Y, Zhou B, Zhou ZG and Sun XF: Downregulation of microRNA-124 is an independent prognostic factor in patients with colorectal cancer. Int J Colorectal Dis 28: 183-189, 2013.

13. Lewis BP, Burge CB and Bartel DP: Conserved seed pairing, often flanked by adenosines, indicates that thousands of human genes are microRNA targets. Cell 120: 15-20, 2005.

14. Deng X, Ma L, Wu M, Zhang G, Jin C, Guo Y and Liu R: miR-124 radiosensitizes human glioma cells by targeting CDK4. J Neurooncol 114: 263-274, 2013.

15. Vecchione A, Belletti B, Lovat F, Volinia S, Chiappetta G, Giglio S, Sonego M, Cirombella R, Onesti EC, Pellegrini P, et al: A microRNA signature defines chemoresistance in ovarian cancer through modulation of angiogenesis. Proc Natl Acad Sci USA 110: 9845-9850, 2013

16. Aigner A: MicroRNAs (miRNAs) in cancer invasion and metastasis: Therapeutic approaches based on metastasis-related miRNAs. J Mol Med (Berl) 89: 445-457, 2011.

17. Farazi TA, Hoell JI, Morozov P and Tuschl T: MicroRNAs in human cancer. Adv Exp Med Biol 774: 1-20, 2013.

18. Di Leva G and Croce CM: The Role of microRNAs in the tumorigenesis of ovarian cancer. Front Oncol 3: 153, 2013.

19. Fang L, Deng Z, Shatseva T, Yang J, Peng C, Du WW, Yee AJ, Ang LC, He C, Shan SW and Yang BB: MicroRNA miR-93 promotes tumor growth and angiogenesis by targeting integrin- $\beta 8$. Oncogene 30: 806-821, 2011.

20. Su Y, Li X, Ji W, Sun B, Xu C, Li Z, Qian G and Su C: Small molecule with big role: MicroRNAs in cancer metastatic microenvironments. Cancer Lett 344: 147-156, 2014.

21. Garzon R, Calin GA and Croce CM: MicroRNAs in cancer. Annu Rev Med 60: 167-179, 2009.

22. Zhang Z, Zheng W and Hai J: MicroRNA-148b expression is decreased in hepatocellular carcinoma and associated with prognosis. Med Oncol 31: 984, 2014.

23. Bartel DP: MicroRNAs: Genomics, biogenesis, mechanism and function. Cell 116: 281-297, 2004.

24. Schwabe RF and Wang TC: Targeting liver cancer: First steps toward a miRacle? Cancer Cell 20: 698-699, 2011.

25. Iorio MV, Casalini P, Piovan C, Braccioli L and Tagliabue E: Breast cancer and microRNAs: Therapeutic impact. Breast 20 (Suppl 3): S63-S70, 2011.

26. Chen Y, Song Y, Wang Z, Yue Z, Xu H, Xing C and Liu Z: Altered expression of MiR-148a and MiR-152 in gastrointestinal cancers and its clinical significance. J Gastrointest Surg 14: 1170-1179, 2010.

27. Chen Y, Song YX and Wang ZN: The microRNA-148/152 family: Multi-faceted players. Mol Cancer 12: 43, 2013.

28. Song Y, Xu Y, Wang Z, Chen Y, Yue Z, Gao P, Xing C and $\mathrm{Xu}$ H: MicroRNA-148b suppresses cell growth by targeting cholecystokinin-2 receptor in colorectal cancer. Int J Cancer 131: 1042-1051, 2012

29. Song YX, Yue ZY, Wang ZN, Xu YY, Luo Y, Xu HM, Zhang X, Jiang L, Xing CZ and Zhang Y: MicroRNA-148b is frequently down-regulated in gastric cancer and acts as a tumor suppressor by inhibiting cell proliferation. Mol Cancer 10: 1, 2011.

30. Bloomston M, Frankel WL, Petrocca F, Volinia S, Alder H, Hagan JP, Liu CG, Bhatt D, Taccioli C and Croce CM: MicroRNA expression patterns to differentiate pancreatic adenocarcinoma from normal pancreas and chronic pancreatitis. JAMA 297: 1901-1908, 2007. 
31. Schetter AJ, Leung SY, Sohn JJ, Zanetti KA, Bowman ED, Yanaihara N, Yuen ST, Chan TL, Kwong DL, Au GK, et al: MicroRNA expression profiles associated with prognosis and therapeutic outcome in colon adenocarcinoma. JAMA 299: 425-436, 2008

32. Yu T, Wang XY, Gong RG, Li A, Yang S, Cao YT, Wen YM, Wang CM and Yi XZ: The expression profile of microRNAs in a model of 7,12-dimethyl-benz[a]anthrance-induced oral carcinogenesis in Syrian hamster. J Exp Clin Cancer Res 28: 64, 2009.

33. Cimino D, De Pittá C, Orso F, Zampini M, Casara S, Penna E, Quaglino E, Forni M, Damasco C, Pinatel E, et al: miR148b is a major coordinator of breast cancer progression in a relapse-associated microRNA signature by targeting ITGA5, ROCK1, PIK3CA, NRAS and CSF1. FASEB J 27: 1223-1235, 2013

34. Wu Y, Liu GL, Liu SH, Wang CX, Xu YL, Ying Y and Mao P: MicroRNA-148b enhances the radiosensitivity of non-Hodgkin's lymphoma cells by promoting radiation-induced apoptosis. J Radiat Res 53: 516-525, 2012.

35. Zheng B, Liang L, Wang C, Huang S, Cao X, Zha R, Liu L, Jia D, Tian Q, Wu J, et al: MicroRNA-148a suppresses tumor cell invasion and metastasis by downregulating ROCK1 in gastric cancer. Clin Cancer Res 17: 7574-7583, 2011.

36. Nakagawa H, Yoshioka K, Miyahara E, Fukushima Y, Tamura M and Itoh K: Intrathecal administration of Y-27632, a specific rho-associated kinase inhibitor, for rat neoplastic meningitis. Mol Cancer Res 3: 425-433, 2005.

37. Xue F, Takahara T, Yata Y, Xia Q, Nonome K, Shinno E, Kanayama M, Takahara S and Sugiyama T: Blockade of Rho/Rho-associated coiled coil-forming kinase signaling can prevent progression of hepatocellular carcinoma in matrix metalloproteinase-dependent manner. Hepatol Res 38: 810-817, 2008.

38. Lane J, Martin TA, Watkins G, Mansel RE and Jiang WG: The expression and prognostic value of ROCKI and ROCKII and their role in human breast cancer. Int J Oncol 33: 585-593, 2008.

39. Vishnubhotla R, Sun S, Huq J, Bulic M, Ramesh A, Guzman G, Cho $\mathrm{M}$ and Glover SC: ROCK-II mediates colon cancer invasion via regulation of MMP-2 and MMP-13 at the site of invadopodia as revealed by multiphoton imaging. Lab Invest 87: 1149-1158, 2007.
40. Riento K and Ridley AJ: Rocks: Multifunctional kinases in cell behaviour. Nat Rev Mol Cell Biol 4: 446-456, 2003.

41. Nakagawa O, Fujisawa K, Ishizaki T, Saito Y, Nakao K and Narumiya S: ROCK-I and ROCK-II, two isoforms of Rho-associated coiled-coil forming protein serine/threonine kinase in mice. FEBS Lett 392: 189-193, 1996.

42. Lock FE, Ryan KR, Poulter NS, Parsons M and Hotchin NA: Differential regulation of adhesion complex turnover by ROCK1 and ROCK2. PLoS One 7: e31423, 2012.

43. Zhou X, Wei M and Wang W: MicroRNA-340 suppresses osteosarcoma tumor growth and metastasis by directly targeting ROCK1. Biochem Biophys Res Commun 437: 653-658, 2013.

44. Ma W, Wong CC, Tung EK, Wong CM and Ng IO: RhoE is frequently down-regulated in hepatocellular carcinoma (HCC) and suppresses HCC invasion through antagonizing the Rho/Rho-kinase/myosin phosphatase target pathway. Hepatology 57: 152-161, 2013.

45. An L, Liu Y, Wu A and Guan Y: microRNA-124 inhibits migration and invasion by down-regulating ROCK1 in glioma. PLoS One 8: e69478, 2013.

46. Xu X, Li S, Lin Y, Chen H, Hu Z, Mao Y, Xu X, Wu J, Zhu Y, Zheng $X$, et al: MicroRNA-124-3p inhibits cell migration and invasion in bladder cancer cells by targeting ROCK1. J Transl Med 11: 276, 2013.

47. Ueno K, Hirata H, Shahryari V, Chen Y, Zaman MS, Singh K, Tabatabai ZL, Hinoda Y and Dahiya R: Tumour suppressor microRNA-584 directly targets oncogene Rock-1 and decreases invasion ability in human clear cell renal cell carcinoma. Br J Cancer 104: 308-315, 2011.

48. Lynch J, Fay J, Meehan M, Bryan K, Watters KM, Murphy DM and Stallings RL: MiRNA-335 suppresses neuroblastoma cell invasiveness by direct targeting of multiple genes from the non-canonical TGF- $\beta$ signalling pathway. Carcinogenesis 33 976-985, 2012

49. Lin SL, Chiang A, Chang D and Ying SY: Loss of mir-146a function in hormone-refractory prostate cancer. RNA 14: 417-424, 2008 